\title{
Multi-objective Evolutionary Feature Selection
}

\author{
Partha Pratim Kundu and Sushmita Mitra \\ Machine Intelligence Unit, Indian Statistical Institute, Kolkata-700 108, India \\ \{ppkundu_r, sushmita\}@isical.ac.in
}

\begin{abstract}
A new method of evolutionary feature selection, using multiobjective optimization in terms of fuzzy proximity and feature set cardinality, is developed. Results on two datasets indicate selection of the correct feature subset.
\end{abstract}

Keywords: Feature selection, Multi-objective optimization, Proximity.

\section{Introduction}

Feature selection is helpful as a preprocessing step for selecting a subset of $n^{\prime}$ features from an original set of $n$ features $\left(n^{\prime}<n\right)$, so that the feature space is optimally reduced according to an evaluation criterion. The use of soft computing is an interesting proposition along this direction [1. We can utilize the search potential of genetic algorithms for efficiently traversing large search spaces. When there are two or more conflicting characteristics to be optimized, often the singleobjective genetic algorithm (GA) 22 requires an appropriate formulation of the single fitness function in terms of an additive combination of the different criteria involved. In such cases multi-objective GAs (MOGAs) 3] provide an alternative, more efficient, approach to search for optimal solutions.

For a dataset with $N$ input patterns we can define an $N \times N$ symmetry matrix, termed the proximity matrix $P$, whose $(i, j)$ th entry represents the similarity (or dissimilarity) measure for the $i$ th and $j$ th patterns for $i, j=1, \ldots, N$. Typically distance functions are used for the purpose. The proximity matrix is a pertinent construct that allows us to deal with structural information inherent in the data. In the fuzzy perspective the concept of similarity boils down to the membership values.

In this article we focus on the proximity relationship from the fuzzy viewpoint, and employ this as one of the fitness functions of the MOGA for evaluating the fitness of the feature subsets of varying cardinality. The use of fuzziness allows us to efficiently model uncertainties and ambiguities inherent in real life overlapping data. The proximity of a pair of patterns in the original feature space is compared with that in the reduced subspace of selected features. If they are similar then this implies that the eliminated feature(s) are not so relevant to the decision making process. The second criterion is the cardinality of the selected feature subset. This is sought to be minimized. A close observation reveals that these two criteria are of a conflicting nature. A smaller subset of features is likely to result in a reduced proximity, and hence reduced classification accuracy (as compared

S. Chaudhury et al. (Eds.): PReMI 2009, LNCS 5909, pp. 74-79 2009.

(C) Springer-Verlag Berlin Heidelberg 2009 
to the original feature space). This prompted us to formulate the problem in the framework of MOGA. The algorithm terminates when an optimal subset of features is obtained, according to the fitness criteria of the multi-objective genetic optimization.

\section{Proximity-Based Feature Selection}

Fuzzy $c$-means (FCM) clusters a set of $N$ patterns $\left\{\boldsymbol{x}_{j}\right\}$ into $c$ clusters by minimizing the objective function $J=\sum_{j=1}^{N} \sum_{i=1}^{c}\left(\mu_{i j}\right)^{m^{\prime}}\left\|\boldsymbol{x}_{j}-\boldsymbol{m}_{i}\right\|^{2}$, where $1 \leq m^{\prime}<\infty$ is the fuzzifier, $\mu_{i j} \in[0,1]$ is the membership of the $j$ th pattern to the $i$ th mean $\boldsymbol{m}_{i}$, and $\|$.$\| is the distance norm, such that \boldsymbol{m}_{i}=\frac{\sum_{j=1}^{N}\left(\mu_{i j}\right)^{m^{\prime}} \boldsymbol{x}_{j}}{\sum_{j=1}^{N}\left(\mu_{i j}\right)^{m^{\prime}}}$ and $\mu_{i j}=\frac{1}{\sum_{k=1}^{c}\left(\frac{d_{j i}}{d_{j k}}\right)^{\frac{2}{m^{\prime}-1}}}, \forall i$, with $d_{j i}=\left\|\boldsymbol{x}_{j}-\boldsymbol{m}_{i}\right\|_{2}$, subject to $\sum_{i=1}^{c} \mu_{i k}=1$, $\forall k$, and $0<\sum_{k=1}^{N} \mu_{i k}<N, \forall i$. The fuzzy partitions computed by FCM, are directly related to the proximity relation. We have

$$
p\left(k_{1}, k_{2}\right)=\sum_{i=1}^{c}\left(\mu_{i k_{1}} \wedge \mu_{i k_{2}}\right),
$$

where $\wedge$ indicates the minimum operation, $p\left(k_{1}, k_{2}\right) \in[0,1]$, and $k_{1}, k_{2}=1, \ldots, N$. Evidently, $p\left(k_{1}, k_{2}\right)=1$ for $k_{1}=k_{2}$ and $p\left(k_{1}, k_{2}\right)=p\left(k_{2}, k_{1}\right)$.

Let there be $K$ subsets of data located in different feature subspaces, with the number of patterns in each subspace being equal to $N$. We form a $c \times N$ partition matrix $\Omega$ consisting of fuzzy membership values $\mu_{i j}$. Note that the dimensionality $n$ of the patterns in each subset could be different. However, the distance of a pattern is computed from the fuzzy cluster prototypes over the same subset of features.

The partition matrix is used to evaluate proximity $p$, which measures the extent to which a pair of patterns are regarded as similar or dissimilar in different subspaces 4. The proximity matrix $P$ contains the proximity results for all possible pairs of patterns.

\subsection{Proximity between Feature Spaces}

Let the cardinality of the original and reduced feature spaces be $n$ and $n^{\prime}$, respectively. Let the proximity matrices in these two spaces be denoted by $P$ and $P^{\prime}$ respectively. The similarity between the two matrices is represented by a scalar value

$$
P_{s}=\sum_{k_{1}=1, k_{2}=1, k_{2}>k_{1}}^{N}\left[p\left(k_{1}, k_{2}\right) \wedge p^{\prime}\left(k_{1}, k_{2}\right)\right],
$$

where $p^{\prime}\left(k_{1}, k_{2}\right)$ is computed by eqn. (1) in the reduced feature space. In order to enhance contrast we use

$$
P_{s_{0}}=\sum_{k_{1}=1, k_{2}=1, k_{2}>k_{1}}^{N}\left[\left(p\left(k_{1}, k_{2}\right) \geq 0.5\right) \wedge\left(p^{\prime}\left(k_{1}, k_{2}\right) \geq 0.5\right)\right] .
$$




\subsection{Use of MOGA}

We encode the problem as a real-coded string of length $L$, with the first $n$ bits corresponding to the $n$ features in the original space. In the bit representation, here a " 1 " implies that the corresponding attribute is present while " 0 " indicates that it is not. Let the size of a chromosome be $L=n+c \times n=n \times(c+1)$. The $c$ cluster centers are encoded in real form in the subsequent $c \times n$ bits. Only those features of the centers in the second part of the string, corresponding to a "1" in the first part, are considered during clustering. Fig. 1 depicts such an encoding in a chromosome, representing a sample set of cluster prototypes in a feature subspace. Initially all the bits are set randomly.

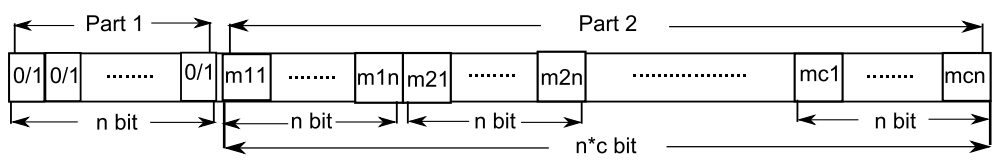

Fig. 1. An encoded chromosome representing a feature subspace and cluster prototypes

The objective is to optimize a conflicting set of requirements i.e., select a minimal number of features that enable us to arrive at an acceptable (classification) decision. We employ MOGA with $P_{s_{0}}$ of eqn. (3) as the fitness function $f_{1}=P_{s_{0}}$. The second fitness function corresponds to the cardinality of the feature set under consideration, and is defined as $f_{2}=n^{\prime}$. While $f_{2}$ gives credit to a candidate string containing less attributes, the function $f_{1}$ determines the extent to which the set of all pairs of patterns belong to the same cluster in the two feature spaces, viz., original and reduced subspace. These two fitness functions are optimized in the framework of MOGA. Clustering is done by FCM to update the prototypes $m_{i}$, in the different subspaces.

The performance of the selected feature subset is validated in terms of its accuracy of performance, as measured by the well-known $k$-nearest neighbour $(k-\mathrm{NN})$ classifier [5].

\subsection{The Algorithm}

1. Initialize the population randomly.

2. Select a pair of chromosomes randomly, along with a random bit location for the initiation of single-point crossover.

3. Perform two-point mutation on the two parts of the string with the corresponding real values being randomly altered. In the first part, the value of the randomly chosen bit (signifying presence or absence of the corresponding attribute) is flipped. In case of the second part, the value $m_{i k_{\text {old }}}$ corresponds to a randomly chosen attribute, $k$ of a $i^{\text {th }}$ cluster center; this is mutated as $m_{i k_{\text {new }}}=\sigma \times x+m_{i k_{\text {old }}}$, where the random variable $x(\sim N(0,1)$ is drawn from a Gaussian distribution, the variance $\sigma^{2}$ determines the magnitude 


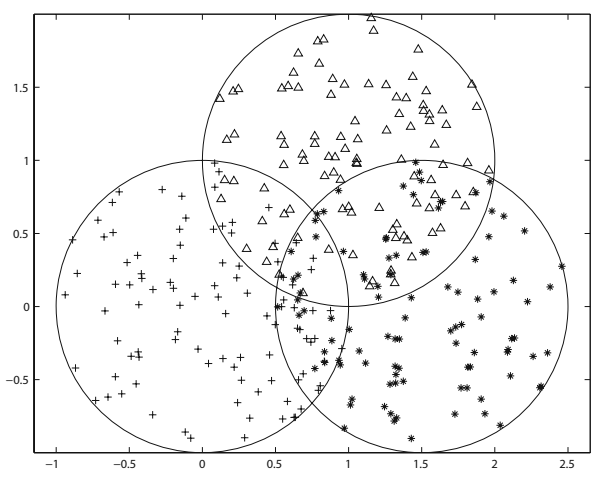

Fig. 2. Synthetic data

of this perturbation at position $m_{i k_{o l d}}$, and $m_{i k_{n e w}}$ is its new value after mutation.

4. Calculate the fitness values using $f_{1}$ and $f_{2}$.

5. Rank the population using dominance criteria.

6. Calculate the crowding distance of the chromosome.

7. Combine parent and offspring population. Replace the parent population by the best members of the combined population.

Note that the cluster centers are set randomly. During crossover and mutation the centers get modified. Their effect is reflected through the proximity function $P_{s o}$ into the fitness evaluation. The features present in a chromosome, as indicated by the " 1 " $\mathrm{s}$ in the first part, determine the feature subspace. They affect the computation of proximity in terms of cluster prototypes, using eqns. (1).

\section{Experimental Results}

The performance of the algorithm was tested on a synthetic data set and Iris flower data. Iris contains 150 instances, with four features and three classes of iris flower. The synthetic data contains three clusters, each with 100 randomly generated patterns. The two-dimensional scatter plot of Fig. 2 depicts the patterns lying within circles of unit radius, each having different centers. A lot of overlapping is artificially introduced. We introduced a third attribute having completely random values, to test the effectiveness of the algorithm in identifying the significance of the first two features.

The performance of the algorithm for strings generated in the non-dominated Pareto front, for the two datasets, are presented in Table 1]. The second column indicates the selected attributes, marked by a " 1 ". The third and fourth columns denote the corresponding two fitness functions, as evaluated by $f_{1}$ and $f_{2}$. The last four columns provide the classification accuracy (for the three classes and the total) for the corresponding feature subspace, corresponding to different values of $k$. 
Table 1. Performance of selected features in Pareto-optimal front

\begin{tabular}{|c|c|c|c|c|c|c|c|c|}
\hline \multirow{3}{*}{ Dataset } & \multirow{3}{*}{$\begin{array}{c}\text { Feature } \\
\text { subspace }\end{array}$} & \multirow{3}{*}{$\begin{array}{c}f_{1} \\
\text { Proximity }\end{array}$} & \multirow{3}{*}{$\begin{array}{c}f_{2} \\
\text { Cardinality }\end{array}$} & \multicolumn{5}{|c|}{$k$-NN performance } \\
\hline & & & & \multirow[t]{2}{*}{$k=$} & \multicolumn{4}{|c|}{ accuracy (\%) } \\
\hline & & & & & C1 & $\mathrm{C} 2$ & \begin{tabular}{|l|} 
C3 \\
\end{tabular} & NET \\
\hline \multirow{12}{*}{ Iris } & \multirow{4}{*}{0111} & \multirow{4}{*}{5801.68} & \multirow{4}{*}{3} & 1 & 100.00 & 94.00 & 90.00 & 94.66 \\
\hline & & & & 3 & 100.00 & 92.00 & 98.00 & 94.00 \\
\hline & & & & 5 & 100.00 & 94.00 & 88.00 & 94.00 \\
\hline & & & & 7 & 100.00 & 94.00 & 94.00 & 96.00 \\
\hline & \multirow{4}{*}{0010} & \multirow{4}{*}{3401.15} & \multirow{4}{*}{1} & 1 & 100.00 & 94.00 & 68.00 & 88.00 \\
\hline & & & & 3 & 100.00 & 88.00 & 86.00 & 91.33 \\
\hline & & & & 5 & 100.00 & 92.00 & 80.00 & 90.67 \\
\hline & & & & 7 & 100.00 & 92.00 & 86.00 & 92.67 \\
\hline & \multirow{4}{*}{0011} & \multirow{4}{*}{3552.53} & \multirow{4}{*}{2} & 1 & 100.00 & 94.00 & 90.00 & 94.66 \\
\hline & & & & 3 & 100.00 & 94.00 & 92.00 & 95.33 \\
\hline & & & & 5 & 100.00 & 96.00 & 92.00 & 96.00 \\
\hline & & & & 7 & 100.00 & 96.00 & 92.00 & 96.00 \\
\hline \multirow{12}{*}{ Synthetic } & \multirow{4}{*}{111} & \multirow{4}{*}{21074.38} & \multirow{4}{*}{3} & 1 & 80.00 & 77.00 & 74.00 & 77.00 \\
\hline & & & & 3 & 78.00 & 76.00 & 72.00 & 75.33 \\
\hline & & & & 5 & 83.00 & 72.00 & 75.00 & 76.67 \\
\hline & & & & 7 & 83.00 & 75.00 & 70.00 & 76.00 \\
\hline & \multirow{4}{*}{100} & \multirow{4}{*}{7444.99} & \multirow{4}{*}{1} & 1 & 75.00 & 66.00 & 52.00 & 64.33 \\
\hline & & & & 3 & 77.00 & 55.00 & 41.00 & 57.67 \\
\hline & & & & 5 & 81.00 & 60.00 & 38.00 & 59.67 \\
\hline & & & & 7 & 87.00 & 64.00 & 35.00 & 62.00 \\
\hline & \multirow{4}{*}{110} & \multirow{4}{*}{15255.10} & \multirow{4}{*}{2} & 1 & 83.00 & 78.00 & 79.00 & 80.00 \\
\hline & & & & 3 & 81.00 & 78.00 & 79.00 & 79.33 \\
\hline & & & & 5 & 84.00 & 81.00 & 82.00 & 82.33 \\
\hline & & & & 7 & 87.00 & 82.00 & 78.00 & 82.33 \\
\hline
\end{tabular}

Table 2. Comparative study on Iris data

\begin{tabular}{|c|c|c|c|c|c|}
\hline Algorithm & $P R$ & $D K$ & $P C$ & $I M$ & $R *$ \\
\hline Selected Features & $\{3,4\}$ & $\{3,4\}$ & $\{3,4\}$ & $\{3,4\}$ & $\{3,4\}$ \\
\hline
\end{tabular}

In case of Iris data, it is observed that the choice of feature 3 occurs in all the three cases, with feature 4 being selected next most frequently. Together they result in the second highest proximity and second lowest cardinality. The accuracy in classification is also the best over all values of $k$. It is well-known that these are the two features most important for discriminating between the classes in this benchmark data. It is to be noted that the accuracy of performance over the original feature space (highest proximity and highest cardinality) turned out to be $95.33 \%, 95.33 \%, 96.00 \%, 96.00 \%$, respectively, for $k=1,3,5,7$. Interestingly, with $k=3,5,7$, the performance in the reduced space is found to be equally good - inspite of the elimination of two features.

The result obtained by the proposed algorithm (model PR) for Iris data was compared with some of the existing techniques, considered as benchmarks in this study. These are the statistical method of Devijver and Kittler [5] (model $\mathrm{DK}$ ), the fuzzy entropy based method of Pal and Chakraborty [6] (model PC), the neural network based method of Ruck and Rogers [7] (model $\mathrm{R}^{*}$ ), and that of Ishibuchi [8] (model IM). Table 2 demonstrates a comparative study of the feature subsets selected by different algorithms for the Iris data. The overall study shows that the results tally with each other. The features 4 and 3 are found to be more important than the features 1 and 2 for classifying Iris data. 
We know that the synthetic data is represented with the first two attributes, with the third feature being inserted randomly. As evident from the results, the selection of the first two features (only) results in an improved accuracy, over all values of $k$, due to the elimination of the unimportant third feature.

\section{Conclusion}

A new multi-objective feature selection algorithm has been developed. Fuzzy proximity was used to evaluate the similarity between the original and reduced feature subspaces. The cardinality of the feature subset was simultaneously minimized. Experimental results demonstrated the effectiveness of the developed method.

\section{References}

1. Banerjee, M., Mitra, S., Banka, H.: Evolutionary-rough feature selection in gene expression data. IEEE Trans. Syst. Man Cybern Part C 37, 622-632 (2007)

2. Goldberg, D.E.: Genetic Alogorithm in Search Optimization and Machine Learning. Addison-Wesley, Reading (1989)

3. Deb, K.: Multi-Objective Optimiztion using Evolutionary Algorithms. John Wiley \& Sons, Chichester (2002)

4. Pedrycz, W., Loia, V., Senatore, S.: P-FCM: A proximity-based fuzzy clustering. Fuzzy Sets and Systems 148, 21-41 (2004)

5. Devijver, P.A., Kittler, J.: Pattern Recognition: A Statistical Approach. PrenticeHall, Englewood Cliffs (1982)

6. Pal, S.K., Chakraborty, B.: Fuzzy set theoretic measure for automatic feature evaluation. IEEE Transactions on Systems, Man, and Cybernetics 16, 754-760 (1986)

7. Ruck, D.W., Rogers, S.K., Kabrisky, M.: Feature selection using a multilayer perceptron. Neural Network Computing 20, 40-48 (1990)

8. Ishibuchi, H., Miyazaki, A.: Determination of inspection order for classifying new samples by neural networks. In: Proceedings of IEEE - International Conference on Neural Networks, Orlando, USA, pp. 2907-2910 (1994) 